\title{
Novel variants in CIITA caused type II bare lymphocyte syndrome: A case report
}

\author{
Yi Zhang, ${ }_{1}$ Yufei Xu, ${ }^{1}$ Yanrong Qing, ${ }^{1}$ Cong Han, ${ }^{1}$ Jiayi Zhu, ${ }^{1}$ Tingting Yu, ${ }^{1}$ Lei Yin, ${ }^{2}$ Ruen Yao, ${ }^{1}$ Jian Wang
}

\section{Abstract}

Background: Type II bare lymphocyte syndrome (BLS II) group A is a rare primary severe immunodeficiency caused by defects in CIITA, one of genes encoding transcriptional regulatory factors for MHC II molecules.

Objective: To report a Chinese boy with mutation of CIITA.

Methods: By reviewing the clinical data of the child and performing a literature search of BLS II group A.

Results: The patient was presented with persistent pneumonia, chronic diarrhea, urinary tract infection, rash, failure to thrive and special facial characteristics. The patient carried novel mutations in CIITA (c.1243delC, p.R415fs 2 and c.3226C > T, p.R1076W) which were identified by next-generation sequencing and confirmed by Sanger sequencing.

Conclusion: This study found novel mutations in the CIITA gene of BLS II, which complemented the mutation spectrum and contributed to the diagnosis, treatment, genetic counseling and prenatal diagnosis of BLS II.

Key words: Type II bare lymphocyte syndrome; CIITA gene; novel mutations; MHC II; genetic disorder

\section{From:}

1 Department of Medical Genetics and Molecular Diagnostic

Laboratory, Shanghai Children's Medical Center, Shanghai Jiao Tong University School of Medicine, Shanghai, People's Republic of China

Department of Nephrology, Shanghai Children's Medical Center, Shanghai Jiao Tong University School of Medicine, Shanghai, People's Republic of China

\section{Corresponding author:}

1. Jian Wang

Department of Medical Genetics and Molecular Diagnostic

Laboratory, Shanghai Children's Medical Center,

Shanghai Jiao Tong University School of Medicine,

No.1678 Dongfang Rd, Shanghai 200127, People's Republic of China E-mail: labwangjiang@126.com

2. Ruen Yao

Department of Medical Genetics and Molecular Diagnostic

Laboratory, Shanghai Children's Medical Center,

Shanghai Jiao Tong University School of Medicine,

No.1678 Dongfang Rd, Shanghai 200127, People's Republic of China

E-mail: yaoruen@126.com

\section{List of abbreviations:}

BLS II Type II Bare Lymphocyte Syndrome

MHC II Class II Major Histocompatibility Complex

CIITA Class II Major Histocompatibility Complex Transactivator

IVIg Intravenous Immunoglobulin Therapy

ARDS Acute Respiratory Distress Syndrome

ACMG American College of Medical Genetics

HGMD Human Genome Mutation Database

PST Proline-, Serine-, and Threonine-rich (PST) domain

NLSs Nuclear Localization Sequences
List of abbreviations (Continued):

LRRs Leucine-Rich Repeats

HSCT Hematopoietic Stem Cell Transplantation

TREC T-cell Receptor Excision Circles

\section{Introduction}

Type II bare lymphocyte Syndrome (BLS II) is a kind of severe primary immunodeficiency which is an autosomal recessive rare heredopathia with clinical symptoms including respiratory and gastrointestinal infections, and liver/biliary tract disease, which was first described in 1979. ${ }^{1}$ Abnormalities in transcription factors, which is essential for the initiation of the transcription of class II major histocompatibility complex (MHC II), would contribute to the occurrence of BLS II , with low $\mathrm{CD}^{+}$cell and absent MHC II expression on lymphocytes. ${ }^{2}$ Four sub-groups of BLS II based on defect type can be categorized into group A, B, C, and D. Group A represents BLS II caused by aberrant class II major histocompatibility complex transactivator (CIITA) and makes up about $11 \%$ of all cases of BLS II. ${ }^{3}$

CIITA is encoded by the 27-exon gene CIITA, located on $16 \mathrm{p} 13.13$, encoding a non-DNA-binding transcription factor composed of 1130 amino acids, encoding an N-terminal acidic domain, a proline-, serine-, and threonine-rich (PST) domain, a GTP-binding site, at least four nuclear localization sequences (NLSs) and leucine-rich regions (LRRs) 
which all can bind with factors independently. ${ }^{4}$ Studies provide evidence that these domains probably interact with each other and any mutation could affect transcriptional activity. ${ }^{4}$ CIITA regulates the expression of $\mathrm{MHC}$ class II of $\mathrm{T}$ helper cell, forming the basis of the adaptive immune response. ${ }^{4}$ Hence, CIITA mutation would cause serious damage to the immune system and severe disorders.

In this report, we present a case of BLS II with novel compound heterozygous variants of CIITA to promote awareness of the disease.

\section{Report of case Patient}

Here we report a case of BLS II in a 19-month-old male Chinese patient. The patient was born at full term via cesarean section to non-consanguineous Chinese parents with a birth weight at $3500 \mathrm{~g}$. Before birth, the couple had undergone an ectopic pregnancy. No family history of similar diseases existed. He was first referred to the hospital at the age of 9 months due to week-long diarrhea, five days of rash, and three days of cough. Later, urinary tract infection appeared. Laboratory inspection revealed a positive CMV infection in sputum specimen, bacterial infection in urine specimen and lactose intolerance, together with normal $\mathrm{CD}^{+} \mathrm{T}$ cell, increased levels of $\mathrm{CD}^{+} \mathrm{T}$ cell and $\mathrm{B}$ cell, inverted $\mathrm{CD} 4 / \mathrm{CD} 8$ ratio, decreased IgA and IgG levels and increased IgM levels (Table 1). The patient received symptomatic antibiotic therapy and intravenous immunoglobulin therapy (IVIg). Rash and urinary tract infections alleviated, while diarrhea persisted. Two months later, the boy was admitted again due to severe pneumonia for not receiving advised regular IVIg. The disease developed into acute respiratory distress syndrome (ARDS), followed by respiratory failure and heart failure. Mechanical ventilation, anti-infective treatment, and IVIg were utilized. Review of flow cytometry and humoral immunity showed normal $\mathrm{CD}^{+} \mathrm{T}$ cell, high level of $\mathrm{CD}^{+} \mathrm{T}$ cell and $\mathrm{B}$ lymphocyte.

Table 1. Immunologic characteristics of the patient

\begin{tabular}{|c|c|c|}
\hline & $9 \mathrm{mo}$ & $12 \mathrm{mo}$ \\
\hline $\operatorname{IgG}(\mathrm{g} / \mathrm{L})(4.09-7.03)$ & $<1.37$ & 5.86 \\
\hline $\operatorname{IgA}(\mathrm{g} / \mathrm{L})(0.21-0.47)$ & $<0.065$ & $<0.066$ \\
\hline $\operatorname{IgM}(\mathrm{g} / \mathrm{L})(0.33-0.73)$ & 4.19 & 1.32 \\
\hline lymphocyte (800-4000/ $\mu \mathrm{L}, 20-40 \%)$ & $14000,66.4$ & $10990,82.4$ \\
\hline $\mathrm{CD}^{+}$cell $(700-2100 / \mu \mathrm{L}, 59-84 \%)$ & $9730,69.52$ & $6470,58.9$ \\
\hline $\mathrm{CD}^{+}{ }^{+} \mathrm{CD}^{+}$cell $(300-1400 / \mu \mathrm{L}, 31-60 \%)$ & $1420,10.11$ & $860,7.80$ \\
\hline $\mathrm{CD}^{+}{ }^{+} \mathrm{CD} 8^{+}$cell $(200-900 / \mu \mathrm{L}, 13-38 \%)$ & $7690,54.92$ & $5270,47.95$ \\
\hline 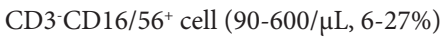 & $460,3.27$ & $160,1.46$ \\
\hline CD3-CD19+ cell (100-500/ $\mu \mathrm{L}, 7-22 \%)$ & $2980,21.28$ & $4000,36.42$ \\
\hline $\mathrm{CD}^{+} / \mathrm{CD}^{+}$ratio $(0.9-3.6)$ & 0.18 & 0.16 \\
\hline CMV-DNA (sputum) & positive & positive \\
\hline
\end{tabular}

Values were obtained at the time of presentation.

Reference ranges are in parentheses.
The level of IgG and IgM returned to normal. CT scan indicates bronchopneumonia and a smaller thymus comparing with normal. Patient recovered discharged but continued to receive regular IVIg therapy for the next 6 months. The patient continued to suffer recurrent lung infections, chronic diarrhea, CMV infection and failure to thrive. In the meantime, IgM level increased again with normal IgG. When the patient reached 19 months, he experienced respiratory failure and heart failure again, but was resuscitated. Sputum culture was positive for Acinetobacter baumannii infection. The immunocyte count and level of immunoglobulin remain abnormal. Further diagnosis and genetic tests were implemented. The child presented with chronic diarrhea (frequency of 3 to 4 times a day) and susceptible to respiratory tract infection. No neurological or physical abnormality found. His weight was 7.3 kilograms (<3 SD) with a head circumference of 44 centimeters $(<2 \mathrm{SD})$ indicating severe malnutrition. He had periocular edema, wide and flat nose bridge, protruding ears, and sparse hair (Figure 1A). Considering the patient's early onset, susceptibleness to multiple infections, abnormal immunocyte and immunoglobulin, the patient might suffer from a genetic disease, so high-throughput sequencing was conducted. The patient is preparing for hematopoietic stem cell transplantation.

\section{CIITA genetic testing result}

Genomic DNA of the patient and his parents was extracted from peripheral blood samples using the Gentra Puregene Blood Kit (Qiagen, Hilden, Germany.) Then next generation sequencing, sanger sequencing, base calling and the sequence read quality assessment were operated. Primers for the amplification of the CIITA gene (GenBank accession No. NM_000246.3) were designed with UCSC ExonPrimer online software. The primers designed for exon 11 were as follows: forward AGTGCTGGCCTTGTGGTG and reverse TTCAAGATGTGGCTGAAAACC. The primers designed for exon 17 18 were as follows: forward GGAAGGCTGACCAT GCAC and reverse CATGATTTGAGCTCCGGG.

Compound heterozygous variants in CIITA (c.1243delC, p.R415fs ${ }^{\star} 2, c .3226 \mathrm{C}>\mathrm{T}$, p.R1076W) were suspected as the possible pathogenic variants through the pipeline described before. Variants were further confirmed using Sanger sequencing in the pedigree. The frameshift variant was detected in the mother at the heterozygous state, located in exon 11 leading to a truncated protein with only 417 amino acids. While the father carried the heterozygous missense variant located in exon 17 on LRRs and caused arginine to change into tryptophan (Figure 1B). Both variants have neither been previously reported and were absent in the Human Genome Mutation Database (HGMD), confirming that the variants are novel.

According to ACMG guideline, the frameshift variant is classified as pathogenic based on evidence PVS1, PM2 and PP4, and the missense mutation is classified as likely pathogenic based on evidence PM1, PM2 and PM3. ${ }^{5}$ The above evidences are explained as follows. Neither are included in control databases including Exome Aggregation Consortium, NHLBI Exome Sequencing Project, 1000 Genomes Project, and the Genome Aggregation Database (PM2). CIITA mutations are known to cause disease in a loss-of-function manner 
A.
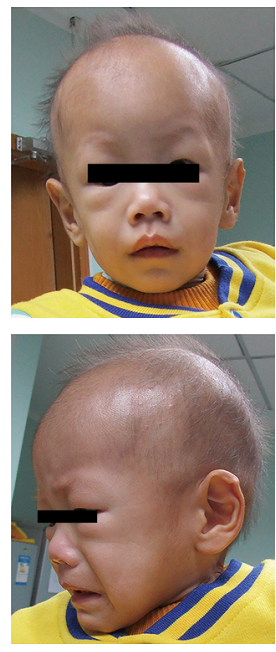

B.

CIITA c.1243delC, p.R415fs*2

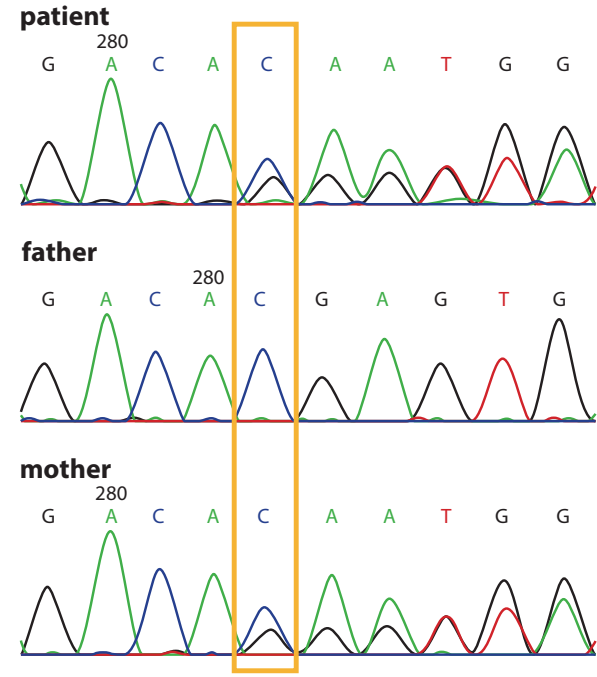

CIITA c.3226C>T, p.R1076W

patient
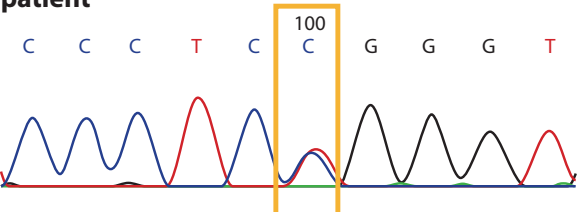

father
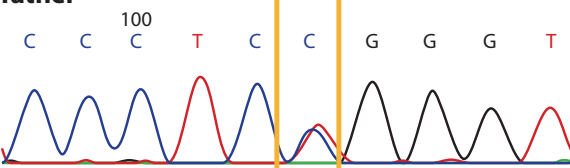

mother
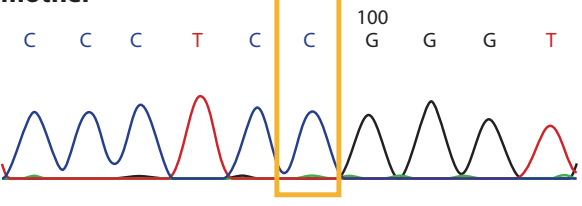

c.2885T>C/p.F962S

c.3004_3084del/p.964_990del

c.3078_3080delCAT/p.1027del1

C.

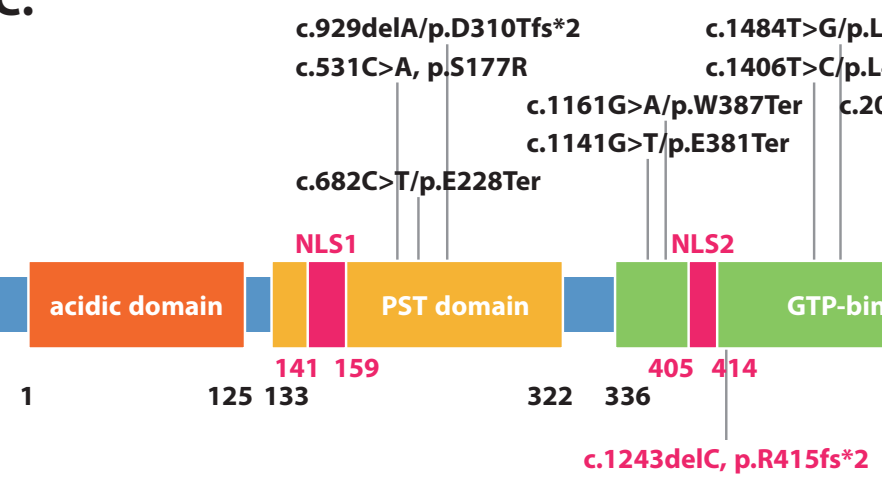

$\begin{array}{ll}\text { c.929delA/p.D310Tfs*2 } & \text { c.1484T }>\text { G/p.L495R } \\ \text { c.531C }>\text { A, p.S177R } & \text { c.1406T }>\text { C/p.L469P }\end{array}$

c.1161G >A/p.W387Ter $\quad$ c.2063G $>$ A/p.W688Ter

c.2408C $>$ A/p.A803V

c.2436C $>A /$ p.C812Ter

c.3136C >G/p.L1046V

c.3265_3348del/p.1050_1078del

c. $3317+1 G>A$

c.1243delC, p.R415fs*2

NLS3

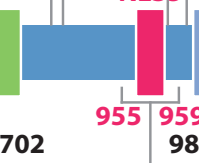

c.2932_3003del/p.940_963del

c.3226C >T, p.R1076W

Figure 1. A. Facial characteristic of the patient. B. Sanger sequencing in CIITA gene. The patient has compound heterozygous mutations in the CIITA gene (c.1243delC, p.R415fs ${ }^{\star 2}$ from her mother; c.3226G $>$ A, p.R1076W from her father). C. Distribution of deleterious mutations ever reported on different domains of CIITA. Mutations found in this article are in magenta.

(PVS1). And LRRs, being functional domain, are mutational hot spots (PM1). With the addition of corresponding clinical phenotype (PP4) and pathogenicity of maternal variant (PM3), a definite genetic diagnosis can be made. ${ }^{5}$ (See Sanger sequencing results in Figure 1B)

\section{Discussion}

BLS II, also known as MHC class II deficiency, is characterized by impaired regulation of the expression of induced and constitutive MHC II. Clinical manifestations of BLS II included early recurrent infections, repeated pneumonia episodes, chronic diarrhea, failure to thrive, and premature death. ${ }^{1}$ The main feature is the loss of MHC II molecules leading to loss of $\mathrm{T}$ lymphocytes, reduced levels of $\mathrm{CD}^{+} \mathrm{T}$ lymphocyte, hypogammaglobulinemia, and impaired antibody production. ${ }^{6}$

Our patient suffered from a failure to thrive, chronic diarrhea, urinary tract infection, recurrent lung infection, severe pneumonia, ARDS, respiratory failure, and heart failure at the age of 9 months. His phenotype is consistent with the clinical manifestation for BLS II. Laboratory tests reflected normal $\mathrm{CD}^{+} \mathrm{T}$ cell, increased $\mathrm{CD}^{+} \mathrm{T}$ cell and $\mathrm{B}$ cell, inverted
$\mathrm{CD}^{+} / \mathrm{CD}^{+}$ratio, reduced levels of $\operatorname{IgA}$ and $\operatorname{IgG}$, increased $\mathrm{IgM}$, and infection with CMV and Acinetobacter baumannii. Following regular antibiotic therapy and IVIg, the level of IgG returned to normal; even though the condition was unstable. Genetic testing was conducted, and the result showed our patient carried novel compound heterozygous variants in CIITA (c.1243delC, p.R415fs ${ }^{\star}$ and c.3226C>T, p.R1076W).

The frameshift variant is classified as pathogenic, affecting the function of CIITA, producing a premature protein without a complete GTP-binding site, NLS, and LRRs. The missense mutation located in the LRRs is classified as likely pathogenic. Although we could not test the expression density of HLA-DR or DQ on B cells or monocytes, the diagnosis of BLS II could be made through a combination of the patient's clinical manifestations and gene mutations.

Based on a review of reported cases, along with a case report in Chinese by Chen et al, there have been only 16 BLS II patients with CIITA mutations (Table SI), including 6 missense, 5 nonsense, 5 deletions and 1 splice site mutation (Figure 1C)..$^{7-19}$ The average onset age was 9.6 months except special cases with no symptoms or extremely late age of onset..$^{7-19}$ 
The immunologic characteristics of the disease have heterogeneity. Normal CD4+ T cell count and high level IgM in our patient are quite distinct but each has been reported with symptoms. ${ }^{7-18}$ There has been only one Chinese patient reported with high level IgM. ${ }^{19}$ Hence our report supplements phenotype spectrum and suggests Chinese patient might be more likely to have increasing immunoglobins.

So far, facial characteristics have only been reported in one case including hypertelorism, and sparse, coarse scalp hair. ${ }^{7}$ Yet, this patient presented with sparse hair, epicanthus, wide and flat nose bridge, nostril anteversion, high columella, deep and flat philtrum, cupid lip, and protruding ears (Figure 1A). This case shows consistent and more detailed facial characteristics. With two in eighteen patients has facial distinction, the fact CIITA mutation might affect the facial appearance might be helpful during clinical diagnosis.

BLS II group A patients showed poor prognosis, with the average age of death being four years. The only possible cure is hematopoietic stem cell transplantation (HSCT) despite only $60 \%$ of success rate. ${ }^{20}$ Thus, acute infection and complications should be actively treated to alleviate relevant clinical symptoms. Intravenous anti-infective drugs, intravenous gamma globulin, parenteral nutrition, and prophylactic use of antibiotics should be taken into consideration as treatment.

In summary, this article is to report a rare case of BLS II with novel pathogenic variants which could enrich the genotypic and phenotypic spectrum for this disorder. A literature review of the previously reported patients revealed more heterogeneous immunologic characteristics and specific facial characteristics that were linked to this rare condition.

\section{Conflict of Interest}

The authors declare no conflict of interest.

\section{Ethics approval and consent to participate}

All procedures performed in studies involving human participants were in accordance with the ethical standards of the institutional and/or national research committee at Shanghai Children's Medical Center (SCMCIRB-Y2019021) and with the 1964 Helsinki declaration and its later amendments or comparable ethical standards. Informed consent was obtained from the parents of the participant included in the study.

\section{Consent for publication}

Written informed consent was obtained from the patient's father for publication of this research.

\section{Availability of data and material}

The datasets (whole-exome sequencing and Sanger sequencing files) used and/or analyzed during the current study are available from the corresponding author on reasonable request.

All data generated or analyzed during this study are included in this published article and its supplementary information files.

\section{Competing interests}

The authors declare that they have no conflict of interests

\section{Authors' contributions}

- YZ critically drafted and revised the manuscript, and reviewed the literature for data on other reported patients suffering from BLS II.

- LY and YX assessed the clinical manifestation of the patient and collected the raw data from our hospital work system.

- JW and RY analysed data generated by next generation sequencing, found the variants, judged the pathogenicity and reviewed the manuscript.

- YQ, CH, JZ and TY operated Sanger sequencing to confirm the variants. All authors have read and approved the manuscript.

\section{Acknowledgments}

We thank all the members of the family for their participation in this study.

\section{Literature review}

All of the literature for previously published CIITA mutations (from 1989 to 2019) was retrieved from PubMed and Human Genome Mutation Database (HGMD), together with a case report in Chinese by Chen et al. (Chen et al., 2018). The clinical characteristics and mutation spectrum of the CIITA gene were then summarized.

\section{Supplementary Material (websites utilized)}

1. 1000 Genomes Project http://www.1000genomes.org

2. Exome Aggregation Consortium http://exac.broadinstitute.org

3. Genome Aggregation Database http://gnomad-old.broadinstitute.org

4. Human Genome Mutation Database http://www.hgmd.cf.ac.uk

5. NHLBI Exome Sequencing Project http://evs.gs.washington.edu/EVS

6. UCSC ExonPrimer http://genome.ucsc.edu/index.html

\section{References}

1. Hanna S, Etzioni A. MHC class I and II deficiencies. J Allergy Clin Immunol. 2014;134(2):269-75.

2. Ouederni M, Vincent QB, Frange P, Touzot F, Scerra S, Bejaoui M, et al. Major histocompatibility complex class II expression deficiency caused by a RFXANK founder mutation: a survey of 35 patients. Blood. 2011;118(19):5108-18.

3. Shrestha D, Szöllosi J, Jenei A. Bare lymphocyte syndrome: an opportunity to discover our immune system. Immunol Lett. 2012;141(2):147-57.

4. Harton JA, Ting JP. Class II transactivator: mastering the art of major histocompatibility complex expression. Mol Cell Biol. 2000;20(17):6185-94.

5. Richards S, Aziz N, Bale S, Bick D, Das S, Gastier-Foster J, et al. Standards and guidelines for the interpretation of sequence variants: a joint consensus recommendation of the American College of Medical Genetics and Genomics and the Association for Molecular Pathology. Genet Med. 2015;17(5):405-24. 
6. Griscelli C, Lisowska-Grospierre B, Mach B. Combined immunodeficiency with defective expression in MHC class II genes. Immunodefic Rev. 1989;1(2):135-53.

7. Dimitrova D, Ong PY, O'Gorman MR, Church JA. Major histocompatibility complex class II deficiency complicated by Mycobacterium avium complex in a boy of mixed ethnicity. J Clin Immunol. 2014;34(6):677-80.

8. Steimle V, Otten LA, Zufferey M, Mach B. Complementation cloning of an MHC class II transactivator mutated in hereditary MHC class II deficiency (or bare lymphocyte syndrome). Cell. 1993;75(1):135-46.

9. Bontron S, Steimle V, Ucla C, Eibl MM, Mach B. Two novel mutations in the MHC class II transactivator CIITA in a second patient from MHC class II deficiency complementation group A. Hum Genet. 1997;99(4):541-6.

10. Quan V, Towey M, Sacks S, Kelly AP. Absence of MHC class II gene expression in a patient with a single amino acid substitution in the class II transactivator protein CIITA. Immunogenetics. 1999;49(11-12):957-63.

11. Peijnenburg A, Van den Berg R, Van Eggermond MJ, Sanal O, Vossen JM, Lennon AM, et al. Defective MHC class II expression in an MHC class II deficiency patient is caused by a novel deletion of a splice donor site in the MHC class II transactivator gene. Immunogenetics. 2000;51(1):42-9.

12. Wiszniewski W, Fondaneche MC, Le Deist F, Kanariou M, Selz F, Brousse $\mathrm{N}$, et al. Mutation in the class II trans-activator leading to a mild immunodeficiency. J Immunol. 2001;167(3):1787-94.

13. Dziembowska M, Fondaneche MC, Vedrenne J, Barbieri G, Wiszniewski W, Picard C, et al. Three novel mutations of the CIITA gene in MHC class II-deficient patients with a severe immunodeficiency. Immunogenetics. 2002;53(10-11):821-9.
14. Ahmed A, Reith W, Puck JM, Cheng LE. Novel Mutation in the Class II Transactivator Associated with Immunodeficiency and Autoimmunity. J Clin Immunol. 2015;35(6):521-2.

15. Yu H, Zhang VW, Stray-Pedersen A, Hanson IC, Forbes LR, de la Morena MT, et al. Rapid molecular diagnostics of severe primary immunodeficiency determined by using targeted next-generation sequencing. J Allergy Clin Immunol. 2016;138(4):1142-51.e2.

16. Al-Mousa H, Abouelhoda M, Monies DM, Al-Tassan N, Al-Ghonaium A, Al-Saud B, et al. Unbiased targeted next-generation sequencing molecular approach for primary immunodeficiency diseases. J Allergy Clin Immunol. 2016;137(6):1780-7.

17. Aluri J, Gupta M, Dalvi A, Mhatre S, Kulkarni M, Hule G, et al. Clinical, Immunological, and Molecular Findings in Five Patients with Major Histocompatibility Complex Class II Deficiency from India. Front Immunol. 2018;9:188.

18. El Hawary RE, Mauracher AA, Meshaal SS, Eldash A, Abd Elaziz DS, Alkady R, et al. MHC-II Deficiency Among Egyptians: Novel Mutations and Unique Phenotypes. J Allergy Clin Immunol Pract. 2019;7(3):856-63.

19. Chen QY, Wang WJ, Sun JQ, Hou J, Ying WJ, Wang XC, et al. MHC classII-deficiency caused by CIITA gene mutation: A report of two cases and literature review. Chinese Journal of Practical Pediatrics. 2018;33(1): 55-9. (In Chinese)

20. Small TN, Qasim W, Friedrich W, Chiesa R, Bleesing JJ, Scurlock A, et al. Alternative donor SCT for the treatment of MHC class II deficiency. Bone Marrow Transplant. 2013; 48(2):226-32. 


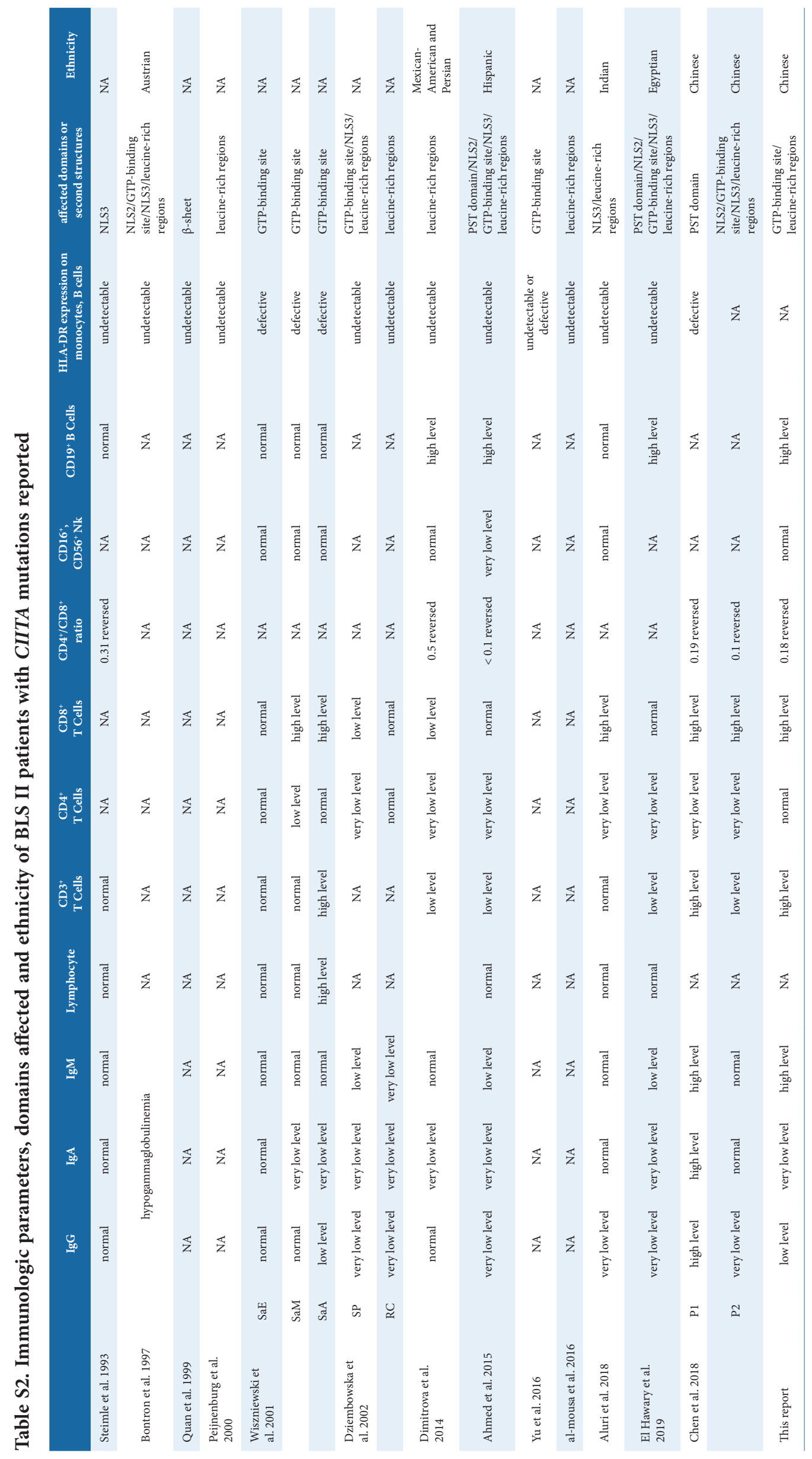

